\title{
Adapted single-cell consensus clustering (adaSC3)
}

\section{Cornelia Fuetterer $^{1}$ (D) Thomas Augustin $^{1}$ (D) $\cdot$ Christiane Fuchs $^{2,3,4}$}

Received: 3 July 2019 / Revised: 11 August 2020 / Accepted: 8 November 2020 /

Published online: 15 December 2020

(c) The Author(s) 2020

\begin{abstract}
The analysis of single-cell RNA sequencing data is of great importance in health research. It challenges data scientists, but has enormous potential in the context of personalized medicine. The clustering of single cells aims to detect different subgroups of cell populations within a patient in a data-driven manner. Some comparison studies denote single-cell consensus clustering (SC3), proposed by Kiselev et al. (Nat Methods 14(5):483-486, 2017), as the best method for classifying single-cell RNA sequencing data. SC3 includes Laplacian eigenmaps and a principal component analysis (PCA). Our proposal of unsupervised adapted single-cell consensus clustering (adaSC3) suggests to replace the linear PCA by diffusion maps, a non-linear method that takes the transition of single cells into account. We investigate the performance of adaSC3 in terms of accuracy on the data sets of the original source of SC3 as well as in a simulation study. A comparison of $\operatorname{adaSC} 3$ with SC3 as well as with related algorithms based on further alternative dimension reduction techniques shows a quite convincing behavior of adaSC3.
\end{abstract}

Keywords Diffusion maps · Non-linear embedding $\cdot$ Single-cell consensus clustering $\cdot$ Simulation data $\cdot$ Single-cell RNA sequencing data

Supplementary Information The online version contains supplementary material available at https://doi. org/10.1007/s11634-020-00428-1.

Cornelia Fuetterer

Cornelia.Fuetterer@stat.uni-muenchen.de

Thomas Augustin

Thomas.Augustin@stat.uni-muenchen.de

Christiane Fuchs

christiane.fuchs@helmholtz-muenchen.de

1 Department of Statistics, Ludwig-Maximilians-University Munich, 80539 Munich, Germany

2 Faculty of Business Administration and Economics, Bielefeld University, 33615 Bielefeld, Germany

3 Institute of Computational Biology, Helmholtz Zentrum Munich, 85764 Neuherberg, Germany

4 Department of Mathematics, Technical University of Munich, 85747 Garching, Germany 
Mathematics Subject Classification 62H30 $\cdot 68 \mathrm{U} 20$

\section{Introduction}

Personalized medicine based on genomic data promises the precise and individualized treatment of diseases using information from a patient's genome (Cho et al. 2012). There is tremendous research interest in this field, especially with regard to cancer. Hereby it is of interest to determine the different stages of cancer as well as the understanding of the complex development of organs for instance, by analyzing the single cells that are obtained from the single-cell RNA sequencing. Data-driven approaches have led to projects such as The Human Cell Atlas (2020), which aims to establish an interpretable structure for the different cell types of single cells and serves as an orientation for the study of diseases. The mission of the Human Cell Atlas is " $(\mathrm{t}) \mathrm{o}$ create comprehensive reference maps of all human cellsthe fundamental units of life-as a basis for both understanding human health and diagnosing, monitoring, and treating disease." Based on the genetic profiles of these single-cell RNA sequencing data, an unsupervised classification allows a data-driven distinction of intra- and intertumoral heterogeneities as well as the determination of different pathways during the development (Duò et al. 2018). The approach of single-cell consensus clustering (SC3) by Kiselev et al. (2017) has gained much attention, not only due to its superior performance in the comparison study of Duò et al. (2018). SC3 is also explicitly tailored to single cell data. Nevertheless, the original incorporation of the linear dimension reduction of principal component analysis may offer a potential for improvement. Following Bendall et al. (2014) and Buettner and Theis (2012), for single cells the transition from one state to another is a non-linear continuous process. Therefore, we propose to replace the PCA of the SC3 method by diffusion maps (Haghverdi et al. 2015), resulting in a new unsupervised algorithm, which we call adapted single-cell consensus clustering (adaSC3). The use of diffusion maps is not only motivated by the biological behavior of single cells but is also supported empirically: First, diffusion maps allow for a natural modeling of the transition of single cells by Markov processes. Secondly, according to Haghverdi et al. (2015), when applying diffusion maps to single cell data, they also seem to perform best compared to other non-linear transformation methods such as independent component analysis, Kernel PCA, Isomap, or Hessian Local Linear Embedding.

Our paper ist structured as follows: We first give an introduction into single-cell RNA sequencing data in Sect. 2. In Sect. 3, we present the methodological background, starting in Sect. 3.1 with the proposed adapted single-cell consensus clustering (adaSC3), as well as some related competing methods. In Sect. 3.2, we focus on the special suitability of diffusion maps, included in our framework of adaSC3. Analyzing some characteristic single-cell RNA sequencing data sets, introduced already in Sect. 2, adaSC3 is compared to its competing methods in Sect. 4. The performance of adaSC3 and its competing methods is further evaluated with partition-wise simulation data in Sect. 5. Section 6 concludes with a brief discussion and outlook. 
Table 1 Characteristics of the scRNA-seq data sets, with $N$ single cells, $G$ genes and $k$ categories of cell types

\begin{tabular}{lrrr}
\hline Data set & \multicolumn{1}{c}{$N$} & \multicolumn{1}{c}{. } & \multicolumn{1}{c}{ (13,322 } \\
\hline Biase et al. (2014) & 49 & 10,333 & 3 \\
Deng et al. (2014) & 269 & 11,154 & 10 \\
Goolam et al. (2016) & 124 & 5757 & 5 \\
Treutlein et al. (2014) & 80 & 10,077 & 5 \\
Yan et al. (2013) & 90 & & 7 \\
\hline
\end{tabular}

\section{Single-cell RNA sequencing data (ScRNA-Seq data)}

The health and development of living organisms can heavily be impacted by the kind and activity of their genes, referred to as gene expression. With the technique of singlecell RNA sequencing (scRNA-seq) introduced by Tang et al. (2009), it is possible to measure the gene expression for single cells. In scRNA-seq, genomic profiles are measured in terms of read counts, that is the number of small sequences ("reads") that result from a cell's RNA that can be identified as belonging to a particular gene. A data set comprising $N$ cells and $G$ genes will hence be a $N \times G$ matrix containing nonnegative integers (including zero). The scRNA-seq data of Biase et al. (2014), Deng et al. (2014), Goolam et al. (2016) and Yan et al. (2013), provided by the Hemberg Group of the Sanger Institute (2020), contain read counts of single cells of a mouse or a human with different cell states that are passed during differential transcription for targeting the analysis of cell division in a pedigree. Gene expression is stochastic, and often the reads follow different distributions for different cell types. Another topic of interest is the examination of having reached varying pathway stages. For example, the experiments of Treutlein et al. (2014) were carried out to investigate cell transition during lung development. In detail, these experiments aim to analyze the development of the distal lung epithelium of the mouse based on the different transcriptional states.

The data sets shown in Table 1 had been used for the evaluation of SC3 by Kiselev et al. (2017). Fulfilling the reproducibility and the sample size criterion for the unsupervised classification leads to the data situation ${ }^{1}$ described in Table 1. Except for the data set of Biase et al. (2014), the distributions of cell types are quite unbalanced encompassing between 80 and 269 single cells.

\footnotetext{
1 Since in the SC3 framework, as described in the transformation step of adaSC3 later, components in higher dimensions are chosen randomly, it is important to focus on data sets with a small amount of single cells, in order to keep the analysis replicable. Moreover, for providing the same data situation as Kiselev et al. (2017), we had to adapt the data set of Biase et al. (2014) such that the number of single cells corresponds to the data description of the original SC3 paper.
} 


\section{Methods}

In this section, we first present the proposed adapted single-cell consensus clustering (adaSC3), which allows, by replacing PCA with diffusion maps, to take the varying pathways and their different transcriptional states into account. Furthermore, we consider several SC3-like approaches that later serve as competitors including different transformation techniques.

In the second part of this section, we look more closely at diffusion maps, which allow an appropriate embedding of the complex data structure of single cells in the transformation step of adaSC3.

\subsection{Unsupervised adapted single-cell consensus clustering (adaSC3) and its competitors}

Single-cell consensus clustering aims at classifying the gene expression of single cells in an unsupervised way such that groups are determined in a data-driven manner for detecting new subgroups or confirming manually determined cell types. The classification process subdivides the cell population with regard to the homogeneity of the genetic profile into subgroups of single cells, which represent, for example, different stages of a disease or of a development process within a patient or within a mouse. The original SC3 is implemented in the software $R$ (R Core Team 2020) and can be described as a pipeline consisting of several transformation steps including an automatic dimension reduction, resulting in a clustering respecting all combinations. For the construction of the adapted single-cell consensus clustering (adaSC3) and its competitors, we rely on the same principle framework as SC3 but consider different transformations. ${ }^{2}$ The concrete procedure of adaSC 3 consists of the following steps:

1. Preprocessing As a result of the scRNA-seq process, one obtains the gene expression matrix $E$ containing the read counts of $N$ single cells and $G$ genes. As a preprocessing step, the original matrix $E$ is reduced by a gene filter, as proposed in the original work, that aims to exclude rare and omnipresent genes. ${ }^{3}$ This leads to the expression matrix $E^{\prime}$ of dimension $N \times G^{\prime}$.

2. Calculation of distance matrix $D$ Based on the expression matrix $E^{\prime}$, the Euclidean distance matrix is constructed for each pairwise single cell combination. Furthermore, two measures of dissimilarity are applied on the log-transformed data of $E^{\prime}$ using the Pearson and the Spearman correlation, respectively. For the sake of simplicity, the obtained distance and dissimilarity matrices will each be referred to as distance matrix $D$.

3. Transformation technique $T$ For each of the obtained distance matrices $D$, we apply Laplacian eigenmaps ${ }^{4}$, introduced by Belkin and Niyogi (2003) as proposed in the original SC3. In addition, we apply diffusion maps, described in more

\footnotetext{
2 AdaSC3 and its competitors are also implemented in $\mathrm{R}$ and are available from the first author upon request.

3 Genes with an expression value of $>2$ in less than $6 \%$ of the cells as well as genes with a positive expression value in more than $94 \%$ of the single cell population are excluded.

4 That are implemented as a spectral embedding in the Python software library Scikit-learn (Pedregosa et al. 2011) as the best size of the neighborhood for the aimed embedding.
} 
detail below, instead of the originally proposed principal component analysis. To each of the six combinations, consisting of three different distance matrices $D$ and two transformation techniques, an eigenvalue decomposition is applied. This leads in total to $3 \times 2=6$ different eigenvalue decompositions, resulting each in respectively $N-1$ eigenvectors $\psi_{1}, \ldots, \psi_{N-1}$ with their ordered eigenvalues $1>\lambda_{1} \geq \cdots \geq \lambda_{N-1}$.

4. Consensus clustering In accordance to SC3, we adopt the automatic selection of the number of eigenvectors to be considered. For each eigenvalue decomposition, a kmeans clustering, with $k$ being deterministic, representing the number of categories of the underlying cell types as proposed in the original paper of Kiselev et al. (2017), is conducted. The automatic selection of eigenvectors of the described scenario starts incorporating the first eigenvector until the rounded integer of the $4 \%$ quantile of the set $\{1, \ldots, N\}$. The subsequent clusterings include each one further eigenvector until the $7 \%$ quantile is reached for including the maximal range of eigenvectors for the last clustering of each combination. The result of each k-means clustering $m$ is summarized in a consensus matrix $\mathcal{C}$, indicating the relative frequency of how often a pair of single cells is grouped together over all $n_{m}$ clusterings. Based on the obtained consensus matrix $\mathcal{C}$, a final completelinkage clustering is performed. It aims to achieve higher performance and a more robust result for the classification of single cells, leading to the final grouping of $k$ subgroups. The quality of clustering is evaluated ex-post by the Adjusted Rand Index (ARI) as proposed in the original work of Kiselev et al. (2017).

Apart from the original SC3 that includes a PCA and Laplacian eigenmaps as transformation techniques, we construct further competing algorithms following the same principle as of adaSC3. Instead of diffusion maps and Laplacian eigenmaps, we propose additional algorithms leading to two different types of constructions, differing in the number of incorporated transformations. The first construction only uses one single transformation technique $T$ in Step 3 of adaSC3. We therefore analyze the influence of the non-linear manifolds of isomaps (IM), locally linear embedding (LLE) as well as the multidimensional scaling (MDS), in addition to the transformation of the principal component analysis (PCA), Laplacian eigenmaps (LE), and diffusion maps (DM), each on their own. ${ }^{5}$ The second type of construction consists of the combination of each mentioned transformation $T$ with Laplacian eigenmaps. This leads to the algorithms named by the incorporated transformations, resulting in LE + IM, LE + LLE and LE + MDS, in addition to the original SC3 (PCA + LE) and adaSC3 (DM+LE).

\subsection{Diffusion maps}

In the following, we describe the motivation of embedding the complex structure of single cells during transition into an appropriate global non-linear manifold, using

\footnotetext{
5 For the construction of IM and LLE, Kayo (2006) proposes to use for IM and LLE the same estimate for the optimal neighborhood size, implemented by the R function calc_k of the R package 1le (Diedrich et al. 2012). Isomaps are then constructed using the $\mathrm{R}$ package vegan (Oksanen et al. 2019); the locally linear embedding further relies on the R package 1le (Diedrich et al. 2012) and MDS is based on the R package stats (R Core Team 2020).
} 
diffusion maps. As stated in the introduction of Angerer et al. (2016), diffusion maps allow the reconstruction of the different states that are connected via different transitions. One possible transition is the mutation of one single cell into another. For the following construction of diffusion maps, we only consider the transition of one single cell into another within one step. Another decisive fact is the robustness of diffusion maps to noise. Furthermore, with the normalization, described in the following, diffusion maps are able to detect lowly represented cell types. Coifman and Lafon (2006) provide the general framework of diffusion maps that can be adapted to single cells following (Angerer et al. 2016) that is presented in the next steps. For the construction of diffusion maps, consider two states $x, y \in \Omega$, with $\Omega$ as the appropriate state space. $x$ and $y$ represent single cells; their gene expressions, measured by count data, lead to the pairwise distance $D(x, y)$.

1. For each choice of the distance measure $D$, each point (single cell) is considered as a node of a symmetric graph with weight function $K_{D}$

$$
K_{D}(x, y)=\exp \left(-\frac{D(x, y)}{2 \alpha^{2}}\right),
$$

indicating the affinity of a pair of single cells with scale parameter $\alpha$, reflecting the best size of the included neighborhood. ${ }^{6}$

2. In the following, we construct the core of a transition kernel of a Markov chain

$$
P_{D}(x, y)=\frac{K_{D}(x, y)}{Z(x)}, \text { with } Z(x)=\sum_{y \in \Omega} K_{D}(x, y) .
$$

3. With a density interpretation of the upper term, the following density normalized transition probability matrix

$$
\tilde{P}_{D}(x, y)=\frac{1}{\tilde{Z}(x)} \frac{K_{D}(x, y)}{Z(x) Z(y)}, \text { with } \tilde{Z}(x)=\sum_{y \in \Omega \backslash x} \frac{K_{D}(x, y)}{Z(x) Z(y)}
$$

can be obtained. As the research question consists of mapping the differentiation behavior of single cells, we are only interested in the transition between single cells. Thus, the diagonal of $\tilde{P}_{D}(x, y)$ is set to zero, and the normalization is adapted appropriately, summing up only the gene expression of differing pairs of single cells with $y \neq x$.

4. Based on the normalized matrix $\tilde{P}_{D}$, indicating the transition of one state to another by an ergodic Markovian diffusion process, the aimed transformation is obtained.

\section{Results}

In this section, we evaluate the clustering performance of adaSC3 and its competitors. The accuracy of combining each of the mentioned transformations in combination with Laplacian eigenmaps is illustrated in Table 2.

\footnotetext{
6 The estimation of $\alpha$ relies on the methods implemented in the R package dest iny (Angerer et al. 2016).
} 
Table 2 ARI of all algorithms including two transformation techniques with *: overall best clustering performance of the combination Laplacian eigenmaps (LE) with isomaps (IM), locally linear embedding (LLE), and multidimensional scaling (MDS), as well as SC3 and adaSC3; bold: best performance comparing SC3 and adaSC3

\begin{tabular}{llllll}
\hline Data Set & LE+ IM & LE + LLE & LE + MDS & SC3 & ada SC3 \\
\hline Biase et al. (2014) & 0.95 & $1.00^{*}$ & $1.00^{*}$ & $\mathbf{0 . 9 5}$ & $\mathbf{0 . 9 5}$ \\
Deng et al. (2014) & 0.68 & 0.70 & 0.56 & 0.67 & $\mathbf{0 . 7 6 *}$ \\
Goolam et al. (2016) & 0.54 & $0.69^{*}$ & 0.54 & $\mathbf{0 . 6 9 *}$ & 0.68 \\
Treutlein et al. (2014) & 0.53 & 0.42 & 0.56 & 0.66 & $\mathbf{0 . 7 7 *}$ \\
Yan et al. (2013) & 0.75 & 0.75 & 0.65 & 0.65 & $\mathbf{0 . 7 5 *}$ \\
\hline
\end{tabular}

AdaSC 3 leads in three out of five cases (Deng et al., Treutlein et al. and Yan et al.) to better clustering results, compared to the original SC3, and it is identical in the case of the data set of Biase et al.. Concerning the competing algorithms, the combinations of LE with IM and MDS tend to deliver worse results compared to ada SC3. However, LE + LLE achieves two times the best performing classification but fails extremely in the case of the Treutlein et al. data set. The slightly worse performance of adaSC compared to SC3 concerning the complete Goolam et al. data set of Table 2 should not be over-interpreted as the resampling results based on leaving out each single cell once, we reach considerably higher performance compared to SC3. Furthermore, we can state that adaSC3 delivers the highest overall performance concerning both the resampling study as well as using only one transformation technique as illustrated in the Supplementary Material. We therefore consider our proposal as generally the best approach among its competitors, of SC3 and its related approaches, based on the benchmarking data sets. This result is especially surprising as the scRNA-seq data sets were originally used for determining the proposed default settings of SC3, such as e.g. the automatic choice for the lower dimension.

\section{Simulation data}

The classification accuracy of the simulation data is investigated in the same way as the scRNA-seq data. We are interested in the consensus clustering accuracy of two simulation groups, which are constructed with different ranges of distribution parameters describing the read counts. With shifted parameter ranges, one can consider the simulation groups as representing a healthy and a diseased population. According to the literature, the use of a zero-inflated negative binomial (ZINB) distribution is recommended as the most adequate approximate distribution for modeling the read counts of single cells. It allows larger variability of read counts compared to the former used Poisson distribution (see e.g. Wagner et al. 2013). Based on the constructed simulation data following a (generalized version of a) ZINB distribution for the expression of each gene, we will investigate the influence of various parameters describing each gene for all possible group partitions for a fixed total number $N$ of single cells. 


\subsection{Construction of simulation data}

The ZINB distribution (Kleiber and Zeileis 2016) is a mixture between a negative binomial probability mass function and a point mass at zero. For generating ZINB distributed simulation data we use the R package emdbook (Bolker B, Bolker Maintainer Ben and Imports, MASS 2020), which is based on a generalization of the negative binomial (NB) distribution with parameters $\mu$ and $\phi$ for the non-zero inflated part. ${ }^{7}$ The parameter $\mu$ is a continuous positive real value, describing the mean. The dispersion parameter $\phi$ represents the shape parameter of the gamma distribution underlying the generalization of the NB. The fraction of zero-inflation is taken into account by the parameter $\pi$.

In the following scenarios, we investigate the influence of different parameters of the distribution family. In order to mimic a realistic situation, the scRNA-seq data of Kolodziejczyk et al. (2015) is taken for estimating the parameters of a ZINB distribution $^{8}$ and allow the construction of ranges for each parameter looking at the shifted quantiles of the estimates of the parameters $\mu$ and $\phi$. This leads to the parameter ranges $\mathcal{M}^{(1)}$ and $\boldsymbol{\Phi}^{(1)}$ for cell population 1 and $\mathcal{M}^{(2)}$ and $\boldsymbol{\Phi}^{(2)}$ for cell population 2. The parameter range $\Pi$ for $\pi$ is set to be the same for both populations. ${ }^{9}$ Thus, the simulated read counts of each gene follow a $\operatorname{ZINB}\left(\mu_{1}, \phi_{1}, \pi_{1}\right)$ distribution for simulation group 1 and a $\operatorname{ZINB}\left(\mu_{2}, \phi_{2}, \pi_{2}\right)$ for simulation group 2 , according to the following scenarios:

- Simulation scenario $(a)$ for different ranges of $\mu$ :

$$
\mu_{1} \in \mathcal{M}^{(1)} \text { and } \mu_{2} \in \mathcal{M}^{(2)}, \phi_{1}=\phi_{2} \in \Phi^{(2)}, \pi_{1}=\pi_{2} \in \Pi
$$

- Simulation scenario $(b)$ for different ranges of $\phi$ :

$$
\mu_{1}=\mu_{2} \in \mathcal{M}^{(2)}, \quad \phi_{1} \in \boldsymbol{\Phi}^{(1)} \text { and } \phi_{2} \in \boldsymbol{\Phi}^{(2)}, \quad \pi_{1}=\pi_{2} \in \Pi
$$

- Simulation scenario (c) for different ranges of $\mu$ and $\phi$ :

$\mu_{1} \in \mathcal{M}^{(1)}$ and $\mu_{2} \in \mathcal{M}^{(2)}, \quad \phi_{1} \in \Phi^{(1)}$ and $\phi_{2} \in \boldsymbol{\Phi}^{(2)}, \pi_{1}=\pi_{2} \in \Pi$

- Simulation scenario $(d)$ for the same range of $\mu$ and $\phi$ :

$$
\mu_{1}=\mu_{2} \in \mathcal{M}^{(2)}, \quad \phi_{1}=\phi_{2} \in \boldsymbol{\Phi}^{(2)}, \quad \pi_{1}=\pi_{2} \in \Pi
$$

For each of the simulation scenarios ( $a$ ) to $(d)$, we sample $N_{1}$ times out of $\operatorname{ZINB}\left(\mu_{1}\right.$ $\left.\phi_{1}, \pi_{1}\right)$ and $N_{2}$ times out of $\operatorname{ZINB}\left(\mu_{2}, \phi_{2}, \pi_{2}\right)$ such that, for comparison purposes, the gene-specific parameters remain the same when generating all possible partitions of $N_{1}: N_{2}$ (with $\left.N_{1}+N_{2}=N\right)$, starting with $1:(N-1)$ until $(N-1): 1$, with $N=50$ for the respective scenario. In order to obtain simulation data with the dimension $N \times G$ for each partition, we repeat this procedure 200 times. Thus, read counts of $G=200$ genes are generated with the new parameter values drawn uniformly from the respective intervals.

\footnotetext{
7 Details explaining the generalization of the negative binomial distribution function based on a mixture of Poisson distributions with gamma distributed Poisson rates can be found e.g. in Fuetterer et al. (2019). They investigate the influence of different heterogeneity degrees of count data using simulation data as well as upand downwardly distorted measurements via the ZINB distribution describing the case of measurements tending to lower read counts and upper read counts.

8 The manual construction of two cell populations rely on the differentially cultured murine embryonic stem cell populations " $2 \mathrm{i}$ " and "serum" for each of the 38.616 genes.

9 The constructed parameter ranges are part of the Supplementary Material.
} 


\subsection{Clustering performance based on simulation data}

The following plots show how the group partition (x-axis) of a combination of two transformation methods influences the clustering accuracy, measured by the Adjusted Rand Index (ARI) (y-axis). In the ideal case, the accuracy is 1 for each of the partitions, which would indicate that the classification perfectly corresponds to the underlying group allocation. This criterion is best met for adaSC3, not only in the case of using only one transformation technique (see Supplementary Material), but also in combination of those with Laplacian eigenmaps (LE) for simulation scenarios $(a)$ to $(c)$. Simulation $(d)$ serves as a reference where no difference in the gene-specific parameters was simulated and no accurate grouping should be detected. Each partition of each scenario is repeated 10 times and the accuracy of the respective clustering results is visualized by boxplots. Results of simulation scenario $(c)$ and $(d)$ can be found in the Supplementary Material.

\subsubsection{Simulation scenario (a): variation in expectation parameter $\mu$}

In the case of differing parameter $\mu$ represented in scenario (a), adaSC3 seems to perform best among the combined methods (see Fig. 1) as well as compared to each method on its own. It can also be seen that the inter quantile range of boxplots have the tendency to be shorter for adaSC 3 and reach higher ARI values compared to its competitors. Therefore, we conclude in general that our approach generates
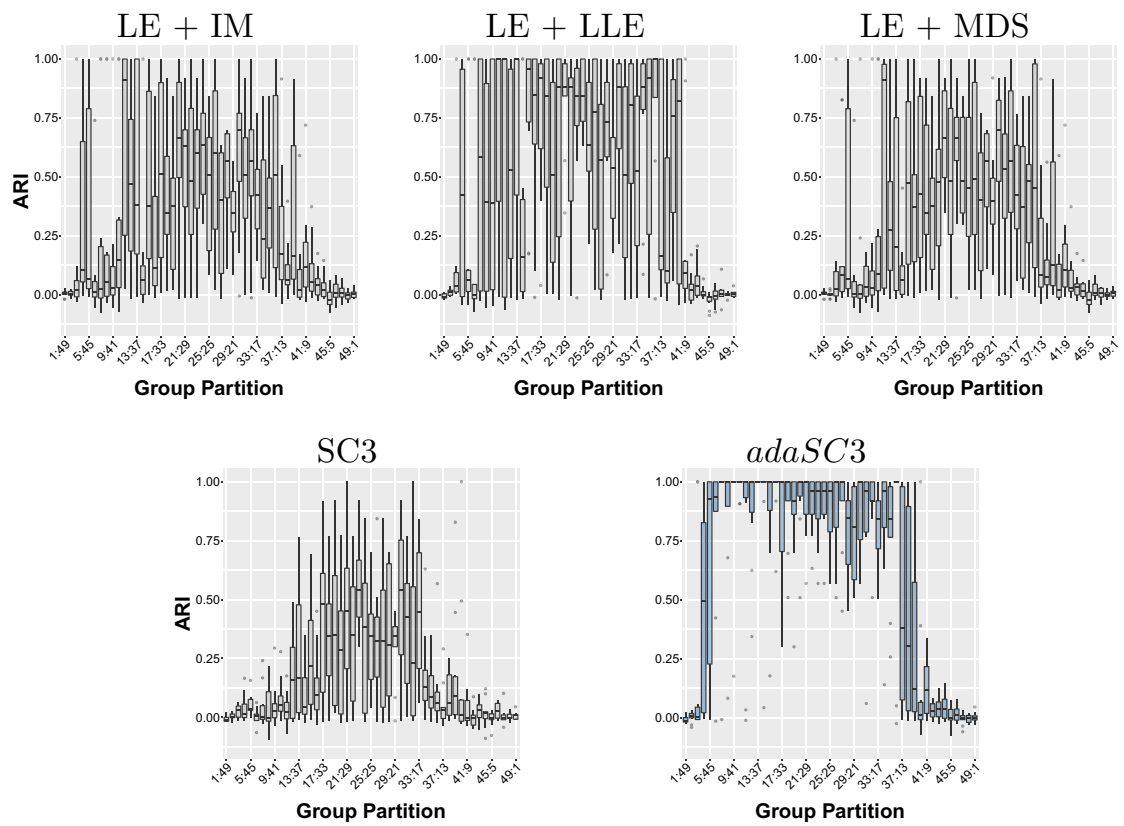

Fig. 1 ARI among all partitions of $N=50$ with regard to the combination of different transformation techniques for simulation scenario $(a)$ 
a more efficient clustering allocation. Furthermore, it should also be noted that the performance of the method adaSC 3 seems to be better when more single cells are part of simulation group 1 compared to simulation group 2. This might allow the interpretation that the detection of true positives achieves higher accuracy compared to the detection of false negatives, given the diseased population has on average higher gene expression.

\subsubsection{Simulation scenario (b): variation in size parameter $\phi$}

For partition-wise created simulation data differing in the parameter $\phi$ one can state, referring to Fig. 2, that apart from adaSC3 the combination of LE + LLE performs quite well, too. However, this method needs more partitions before it starts detecting the difference in the simulation groups and fails drastically earlier compared to adaSC 3 . For approximately balanced data, LE + LLE often leads to worse results. The tendency that the clustering performance depends on the partitions can be confirmed over all methods for simulation scenarios $(a)$ to $(c)$, in which adaSC3 is affected the less.

With regard to scenario $(c)$, the simulated differences of both parameters $\mu$ and $\phi$ lead to a quite accurate classification for most methods with an overall superiority of adaSC 3, representing the scenario being the closest to the reality. For the simulation design with no difference in the simulation groups, the allocation of single cells is as expected and represents random allocation.
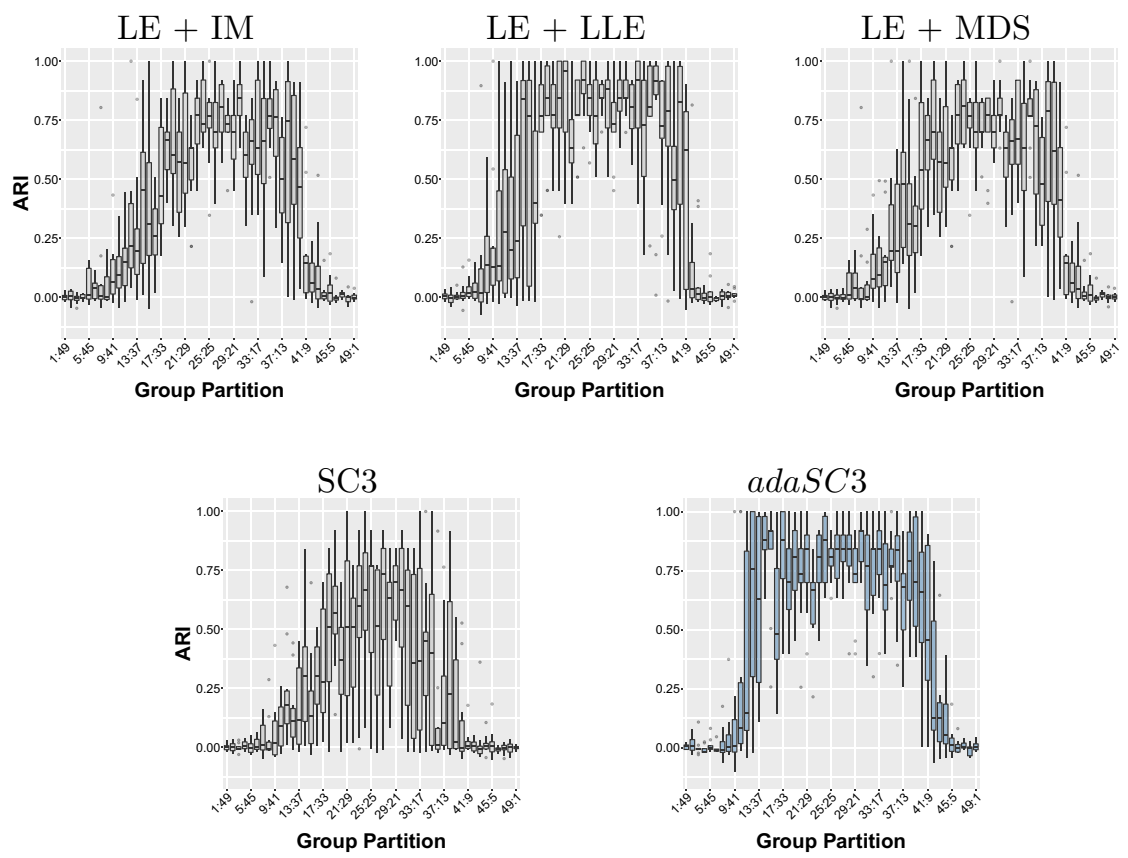

Fig. 2 ARI among all partitions of $N=50$ with regard to the combination of different transformation techniques for simulation scenario $(b)$ 


\section{Conclusions}

The approach of adapted single-cell consensus clustering (adaSC3) is tailored to the clustering of single cells. It reflects the biological structure of single cells by including diffusion maps with the aim to respect the transition process of the underlying data. Indeed, the inclusion of diffusion maps instead of the originally proposed PCA led to a better clustering performance. We consider adaSC 3 to be the best method compared to all investigated competitors, both in the analyzed scRNA-seq data as well as in the simulation study. This motivates further research that takes into account the biological basis of the data before constructing or combining some methods, as this could be rewarded both in terms of interpretation and accuracy, as shown in this paper.

Based on the discovery that balanced data seems to be detected correctly with higher quality, the distribution of classified classes could be taken into account for an unsupervised evaluation. Furthermore, studies of additional scRNA-seq data and further simulations are needed to reinforce the results of this paper. This is especially due to the fact that adaSC3 was evaluated on the same scRNA-seq data used for the development of the original SC3 method. This makes the overall superiority of adaSC3 over SC3 even more surprising, while on the other hand some bias of these data sets favoring SC3-like methods cannot be excluded.

Acknowledgements We are very grateful to the two anonymous referees and the editors for their stimulating and constructive comments. We also want to thank Florian Pfisterer for discussions about diffusion maps. Furthermore, the public data sharing of the scRNA-sequencing data by the Hemberg Group of the Sanger Institute is gratefully acknowledged. In addition, the first author is very thankful to the LMUMentoring program, connecting young researchers with experienced researchers and providing financial support.

Funding Open Access funding enabled and organized by Projekt DEAL.

\section{Compliance with ethical standards}

Conflict of interest The authors declare that they have no conflict of interest.

Open Access This article is licensed under a Creative Commons Attribution 4.0 International License, which permits use, sharing, adaptation, distribution and reproduction in any medium or format, as long as you give appropriate credit to the original author(s) and the source, provide a link to the Creative Commons licence, and indicate if changes were made. The images or other third party material in this article are included in the article's Creative Commons licence, unless indicated otherwise in a credit line to the material. If material is not included in the article's Creative Commons licence and your intended use is not permitted by statutory regulation or exceeds the permitted use, you will need to obtain permission directly from the copyright holder. To view a copy of this licence, visit http://creativecommons.org/licenses/by/4.0/.

\section{References}

Angerer P, Haghverdi L, Büttner M, Theis FJ, Marr C, Buettner F (2016) Destiny: diffusion maps for large-scale single-cell data in R. Bioinformatics 32(8):1241-1243

Belkin M, Niyogi P (2003) Laplacian eigenmaps for dimensionality reduction and data representation. Neural Comput 15(6):1373-1396

Bendall SC, Davis KL, el Amir AD, Tadmor MD, Simonds EF, Chen TJ, Shenfeld DK, Nolan GP, Pe'er D (2014) Single-cell trajectory detection uncovers progression and regulatory coordination in human B cell development. Cell 157(3):714-725 
Biase FH, Cao X, Zhong S (2014) Cell fate inclination within 2-cell and 4-cell mouse embryos revealed by single-cell RNA sequencing. Genome Res 24(11):1787-1796

Bolker B, Bolker Maintainer Ben and Imports, MASS (2020) Package 'emdbook' R package version 1.3.11

Buettner F, Theis FJ (2012) A novel approach for resolving differences in single-cell gene expression patterns from zygote to blastocyst. Bioinformatics 28(18):i626-i632

Cho SH, Jongsu J, Seung IK (2012) Personalized medicine in breast cancer: a systematic review. J Breast Cancer 15(3):265-272

Coifman RR, Lafon S (2006) Diffusion maps. Appl Comput Harmonic Anal 21(1):5-30

Deng Q, Ramsköld D, Reinius B, Sandberg R (2014) Single-cell RNA-seq reveals dynamic, random monoallelic gene expression in mammalian cells. Science 343(6167):193-196

Diedrich H, Abel M., Diedrich Maintainer Holger (2012) Package "lle" R package version 1.1

Duò A, Robinson MD, Soneson C (2018) A systematic performance evaluation of clustering methods for single-cell RNA-seq data. F10000Research 7.2

Fuetterer C, Schollmeyer G, Augustin T (2019) Constructing simulation data with dependency structure for unreliable single-cell RNA-sequencing data using copulas. ISIPTA '19. Proc Mach Learn Res 103:216-224

Goolam M, Scialdone A, Graham SJ, Macaulay IC, Jedrusik A, Hupalowska A et al (2016) Heterogeneity in Oct4 and Sox 2 targets biases cell fate in 4-cell mouse embryos. Cell 165(1):61-74

Haghverdi L, Buettner F, Theis FJ (2015) Diffusion maps for high-dimensional single-cell analysis of differentiation data. Bioinformatics 31(18):2989-2998

Hemberg Group at Sanger Institute (2020) scRNA-Seq Datasets. https://hemberg-lab.github.io/scRNA.seq. datasets/. Accessed 11 Aug 2020

Kayo O (2006) Locally linear embedding algorithm-Extensions and applications. Technical Report, Faculty of Technology, Department of Electrical and Information Engineering, University of Oulo

Kiselev VY, Kirschner K, Schaub MT, Andrews T, Yiu A, Chandra T, Natarajan KN, Rei W, Barahona M, Green AR et al (2017) SC3: consensus clustering of single-cell RNA-Seq data. Nat Methods 14(5):483-486

Kleiber C, Zeileis A (2016) Visualizing count data regressions using rootograms. Am Stat 70(3):296-303

Kolodziejczyk AA, Kim JK, Tsang JCH, Ilicic T, Henriksson J, Natarajan KN, Tuck AC, Gao X, Bühler M, Pentao L, Marioni JC, Teichmann SA (2015) Single cell RNA-sequencing of pluripotent states unlocks modular transcriptional variation. Cell Stem Cell 17(4):471-485

Oksanen J, Blanchet FG, Friendly M, Kindt R, Legendre P, McGlinn D, Minchin PR, O'Hara RB, Simpson GL, Solymos P, Stevens MHH, Szoecs E, Wagner H (2019) vegan: Community Ecology Package. Package 'vegan' R package version 2.5-6

Pedregosa F, Varoquaux G, Gramfort A, Michel V, Thirion B, Grisel O, Blondel M, Prettenhofer P, Weiss R, Dubourg R, Vanderplas J, Passos A, Cournapeau D, Perrot Brucher M, Duchesnay ME (2011) Scikit-learn: machine learning in python. J Mach Learn Res 12:2825-2830

R Core Team (2020) R: a language and environment for statistical computing. R Foundation for Statistical Computing, Vienna, Austria https://www.R-project.org/. Accessed 11 Aug 2020

Tang F, Barbacioru C, Wang Y, Nordman E, Lee C, Xu N, Wang X, Bodeau J, Tuch BB, Siddiqui A, Lao K, Surani MA (2009) MRNA-Seq whole-transcriptome analysis of a single cell. Nat Methods 6:377-382

The Human Cell Atlas. https://www.humancellatlas.org. Accessed 11 Aug 2020

Treutlein B, Brownfield DG, Wu AR, Neff NF, Mantalas GL, Espinoza FH, Desai TJ, Krasnow MA, Quake SR (2014) Reconstructing lineage hierarchies of the distal lung epithelium using single-cell RNA-seq. Nature 509(7500):371-375

Wagner GP, Kin K, Lynch VJ (2013) A model based criterion for gene expression calls using RNA-seq data. Theory Biosci 132(3):159-164

Yan L, Yang M, Guo H, Yang L, Wu J, Li R, Liu P, Lian Y, Zheng X, Yan J et al (2013) Single-cell RNA-Seq profiling of human preimplantation embryos and embryonic stem cells. Nat Struct Mol Biol 20(9):1131

Publisher's Note Springer Nature remains neutral with regard to jurisdictional claims in published maps and institutional affiliations. 